Supporting Information

\title{
Precise Control of the Oxidation State of PbS Quantum Dots Using Rapid Thermal Annealing for Infrared Photodetectors
}

Junyoung Jin ${ }^{1,2}$, Jihoon Kyhm ${ }^{3}$, Do Kyung Hwang ${ }^{4}$, Kyeong-Seok Lee ${ }^{1}$, Tae-Yeon Seong ${ }^{2}$, and Gyu Weon Hwang ${ }^{1, *}$

${ }^{1}$ Center for Electronic Materials Research, Korea Institute of Science and Technology, Seoul 02792, Republic of Korea

${ }^{2}$ Department of Material Science and Engineering, Korea University, Seoul 02841, Republic of Korea

${ }^{3}$ Quantum Functional Semiconductor Research Center, Dongguk University, Joong-gu, Seoul 04620, Republic of Korea

${ }^{4}$ Center for Opto-Electronic Materials and Devices, Korea Institute of Science and Technology, Seoul 02792, Republic of Korea

*Corresponding author email address: gwhwang@kist.re.kr 


\section{Experimental details}

Materials. Lead oxide (99.999\%, PbO), hexamethyldisilathiane, oleic acid (90\%), 1-octadecene $(90 \%)$, tetrabutyl-ammonium iodide (99.0\%, TBAI), (3-mercaptopropyl)tri-methoxysilane (95\%, MPTMS), acetone, $n$-hexane, methanol, octane were purchased from Sigma-Aldrich.

Synthesis of PbS QDs. PbS QDs were synthesized using the previous method with some modifications ${ }^{1}$. Lead oxide ( $4 \mathrm{mmol}, 892 \mathrm{mg}$ ) was added to $22 \mathrm{ml}$ of oleic acid and $44 \mathrm{ml}$ of 1octadecene in a 3-neck flask at room temperature under nitrogen flow. After degassed under vacuum at $100{ }^{\circ} \mathrm{C}$ for $5 \mathrm{~min}$, the solution was then heated to $120{ }^{\circ} \mathrm{C}$ under nitrogen and maintained at $120{ }^{\circ} \mathrm{C}$ for $1 \mathrm{~h}$ to form lead oleate precursor. Hexamethyldisilathiane ( $2 \mathrm{mmol}$ ) dissolved in $6 \mathrm{ml}$ of ODE was swiftly injected into the lead oleate solution at $120{ }^{\circ} \mathrm{C}$. Then, we removed the heating mantle and cooled the flask to room temperature. PbS QDs were dispersed in the solution of octane after purified by acetone. Photoluminescence (PL) and absorbance spectra of the QD solution are shown in Fig. S1. After synthesis, the stock solution was purified two more times by acetone with centrifugation and re-dispersed in octane $(30 \mathrm{mg} / \mathrm{ml})$.

Fabrication of PbS QD photoconductors. Interdigitated Ti $(10 \mathrm{~nm}) / \mathrm{Au}(100 \mathrm{~nm})$ electrodes were deposited by e-beam evaporation on a cleaned $\mathrm{SiO}_{2}(300 \mathrm{~nm}) / \mathrm{Si}$ wafer. Finger spacing and length of the interdigital electrodes are $10 \mu \mathrm{m}$ and $500 \mu \mathrm{m}$, respectively. Then, the substrate was immersed in MPTMS (3 $\mathrm{mM}$ in toluene) for $8 \mathrm{~h}$ to improve the adhesion between the QD film and the substrate. PbS QD films were fabricated by spin-casting followed by a ligand exchange step with TBAI $(10 \mathrm{mg} / \mathrm{ml})$ in methanol and washed 3 times with methanol. This procedure was repeated 10 times to form $350 \mathrm{~nm}$-thick QD films. Finally, the photoconductors were annealed using RTA with tungsten-halogen lamps or a tube furnace in air with different temperature conditions for $30 \mathrm{~s}$ and $20 \mathrm{~min}$ after the temperature reaches $90 \%$ of the set temperature, respectively (Fig. S2). The RTA is set to have $5 \mathrm{sec}$ of the ramp-up time, and the furnace is set to 25 min of the ramp-up time.

Characterization. X-ray photoelectron spectroscopy (XPS) measurements were performed using a Thermo Fisher Scientific Nexsa XPS system, and spectra were analyzed using XPSPEAK 4.1 software. Responsivity and frequency responses were characterized using an 
Agilent 4155C semiconductor parameter analyzer, an HP 33120A function generator, an SR830 lock-in amplifier, and a $1310 \mathrm{~nm}$ laser diode (LD). Transient photocurrent decay measurements were conducted using AFG31000 function generator and TBS1152B oscilloscope. Noise spectral density measurements were performed using an SR570 low-noise preamplifier followed by an Advantest R9211B spectrum analyzer. Absorbance spectra were measured by a PerkinElmer Lambda 750 UV/Vis/NIR spectrophotometer (Fig. S3). PL measurements were carried out using an NIRQuest512 portable spectrometer under $532 \mathrm{~nm}$ illumination.

\section{PbS QD synthesis}

Figure S1(a) and (b) show photoluminescence (PL), absorbance spectra and TEM image of the as-synthesized QDs. The first exciton peak of the QDs is $1391 \mathrm{~nm}$ and the half-width at half maximum (HWHM) of the peak is $48.0 \mathrm{meV}$. PL peak of the QDs is $1451 \mathrm{~nm}$ and the FWHM of the peak is $107.48 \mathrm{meV}$. Average size of the QDs from the TEM image was $5.45 \mathrm{~nm}$ and it is consistent with previous work by Weidman et $\mathrm{al}^{2}$.
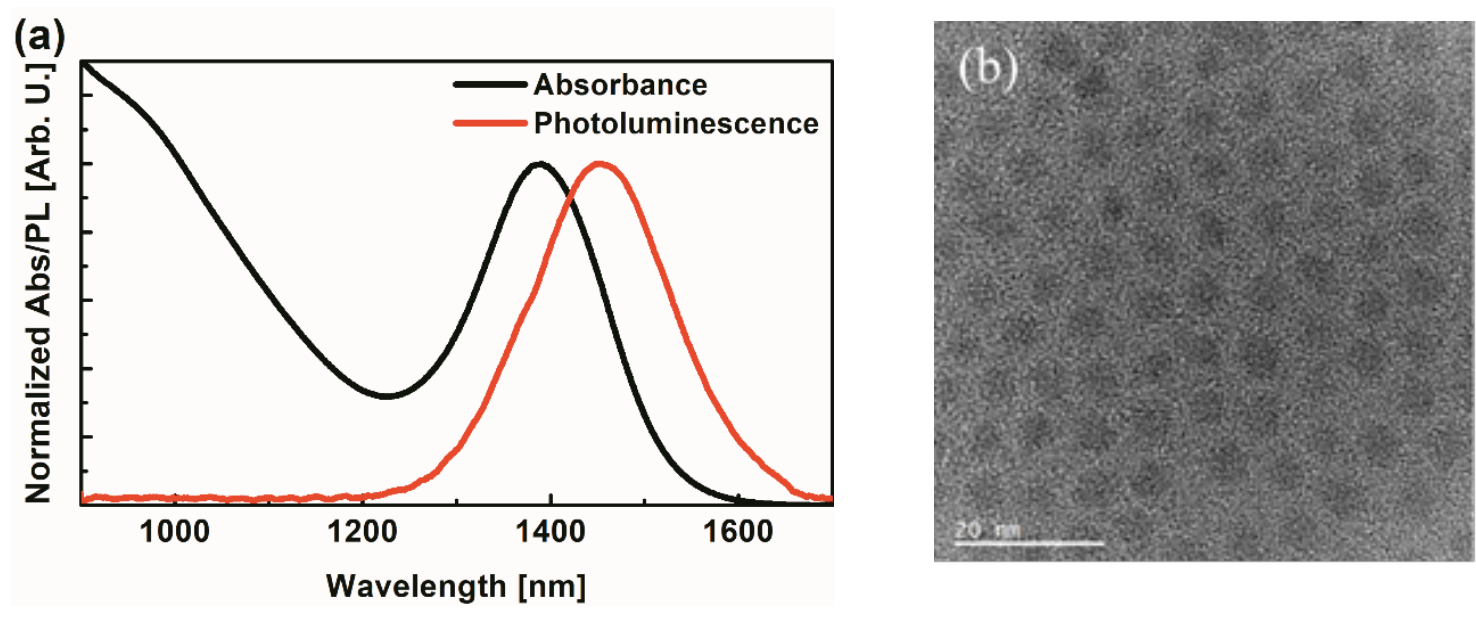

Figure S1. (a) PL and absorbance spectra of the as-synthesized PbS QDs in $\mathrm{C}_{2} \mathrm{Cl}_{4}$ and (b) TEM image of the PbS QDs on a $\mathrm{Cu}$ grid. 


\section{Temperature profiles of the PbS QD photoconductors}
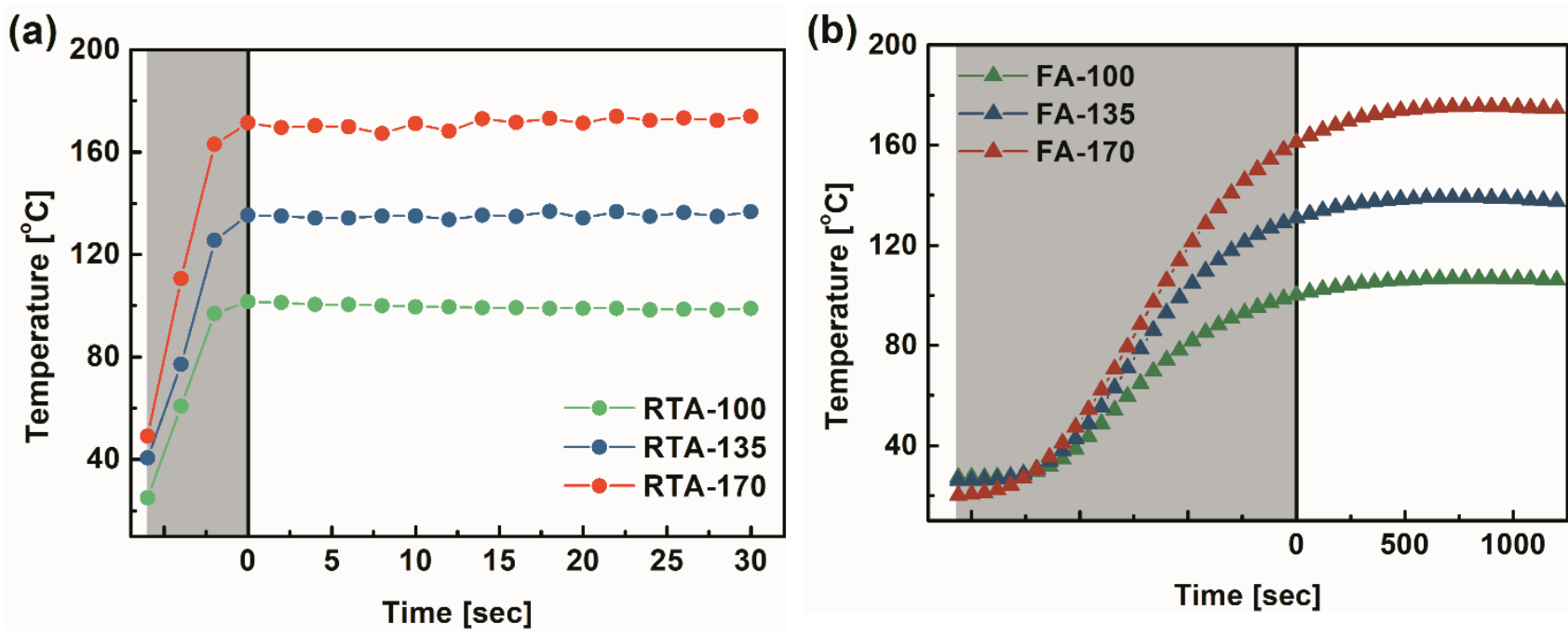

Figure S2. Temperature profiles for the PbS QD photoconductors annealed by (a) RTA and (b) furnace. Gray-colored areas represent ramp-up time.

\section{Absorbance spectra of the annealed QD films}
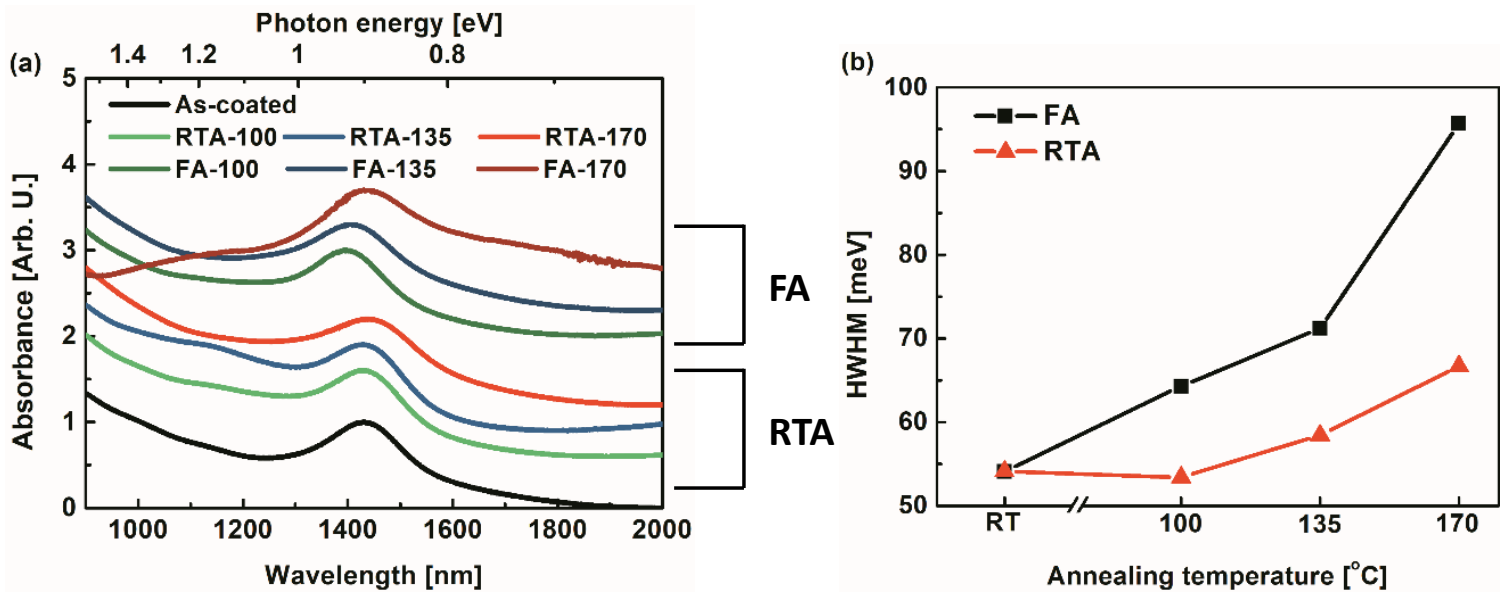

Figure S3. (a) Absorbance spectra of the annealed QD films on glass substrate: Ascoated(black), RTA-100(green), RTA-135(blue), RTA-170(red), FA-100(dark green), FA135(dark blue), FA-170(dark red). (b) The change of the half-width half maxima (HWHMs) of the peaks as a function of annealing temperature. The films annealed with FA broaden wider than the RTA samples as annealing temperature increases. 


\section{The deconvoluted XPS spectra of the PbS QD films}

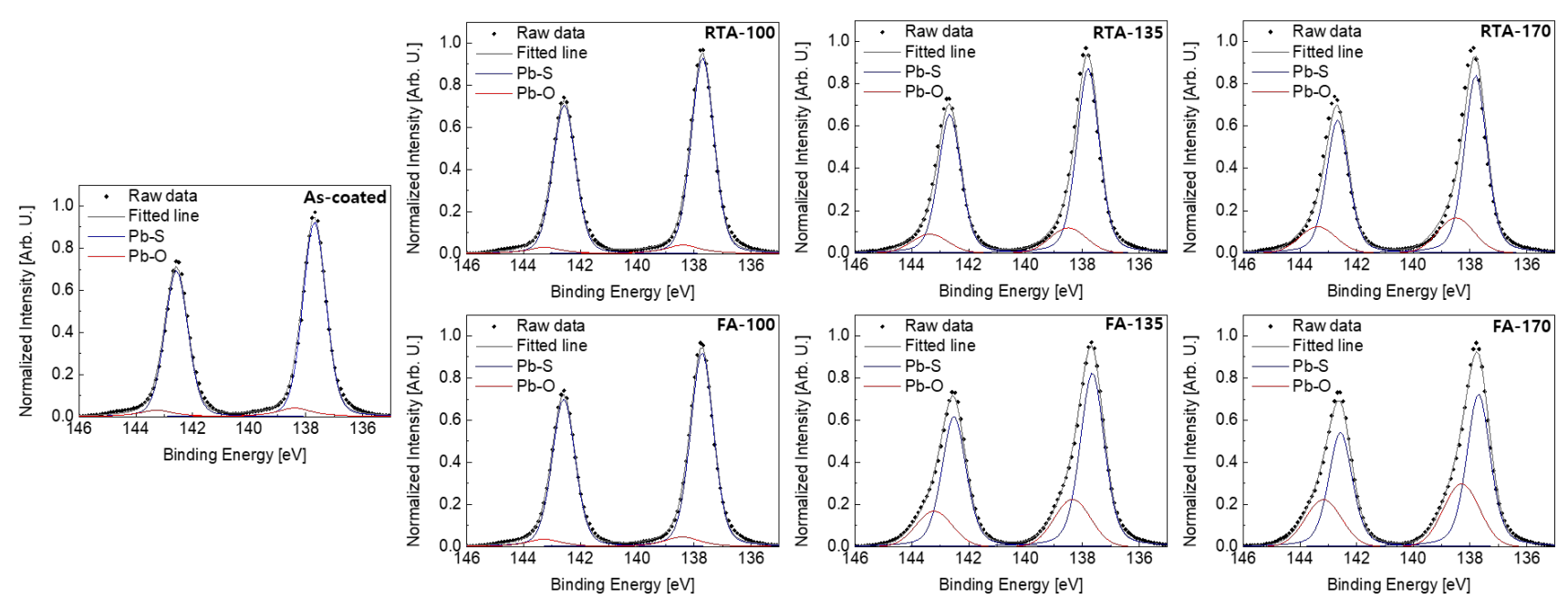

Figure S4. The deconvoluted XPS spectra of the PbS QD films. 


\section{Transient photocurrent decay curves}
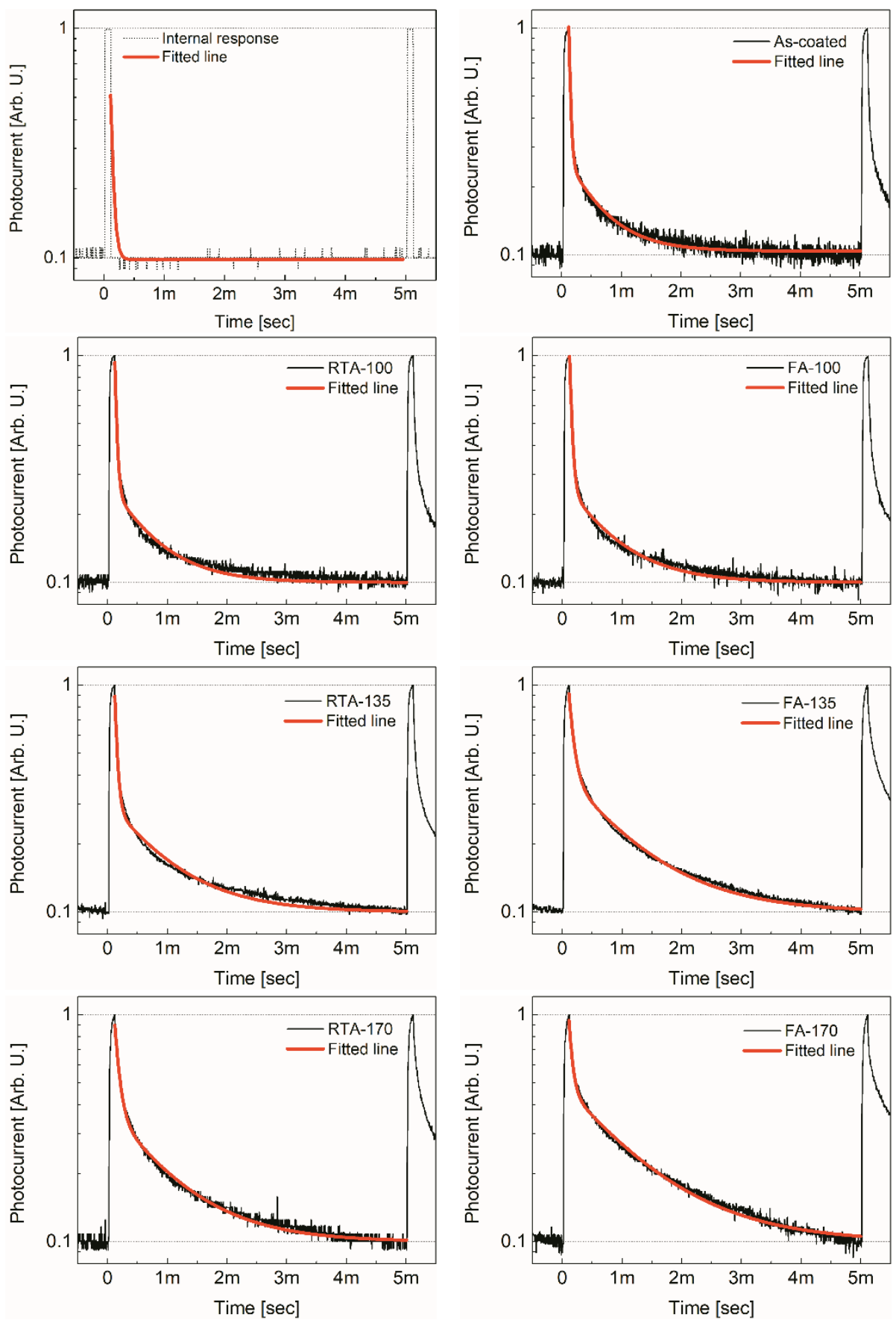

Figure S5. Normalized transient photocurrent decay curves of the PbS QD photoconductors are fitted to bi-exponential function in logarithmic scale. The fast decay component, $\tau_{1}$, is attributed to internal response of measurement system. 
Table S1. Time constant of the fitted curves

\begin{tabular}{ccc}
\hline Sample & $\boldsymbol{\tau}_{\mathbf{1}}[\mathbf{s}]$ & $\boldsymbol{\tau}_{\mathbf{2}}[\mathbf{s}]$ \\
\hline As-coated & $2.77 \times 10^{-5}$ & $5.58 \times 10^{-4}$ \\
RTA-100 & $3.26 \times 10^{-5}$ & $6.75 \times 10^{-4}$ \\
RTA-135 & $4.13 \times 10^{-5}$ & $9.03 \times 10^{-4}$ \\
RTA-170 & $7.82 \times 10^{-5}$ & $9.51 \times 10^{-4}$ \\
FA-100 & $3.91 \times 10^{-5}$ & $7.48 \times 10^{-4}$ \\
FA-135 & $8.27 \times 10^{-5}$ & $1.07 \times 10^{-3}$ \\
FA-170 & $5.92 \times 10^{-5}$ & $1.17 \times 10^{-3}$ \\
\hline
\end{tabular}

\section{Dark current characteristics}

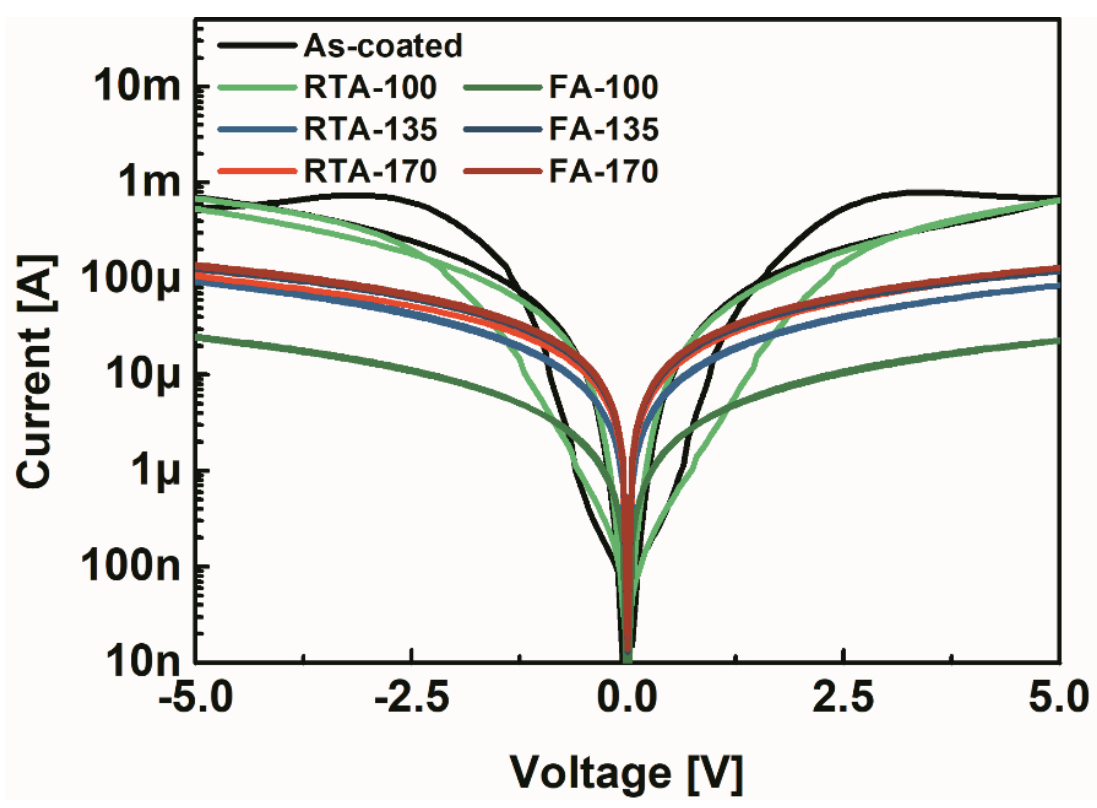

Figure S6. Current-voltage characteristics of the photoconductors in dark. The dark currents affect their noise current spectral density. 


\section{Device image}

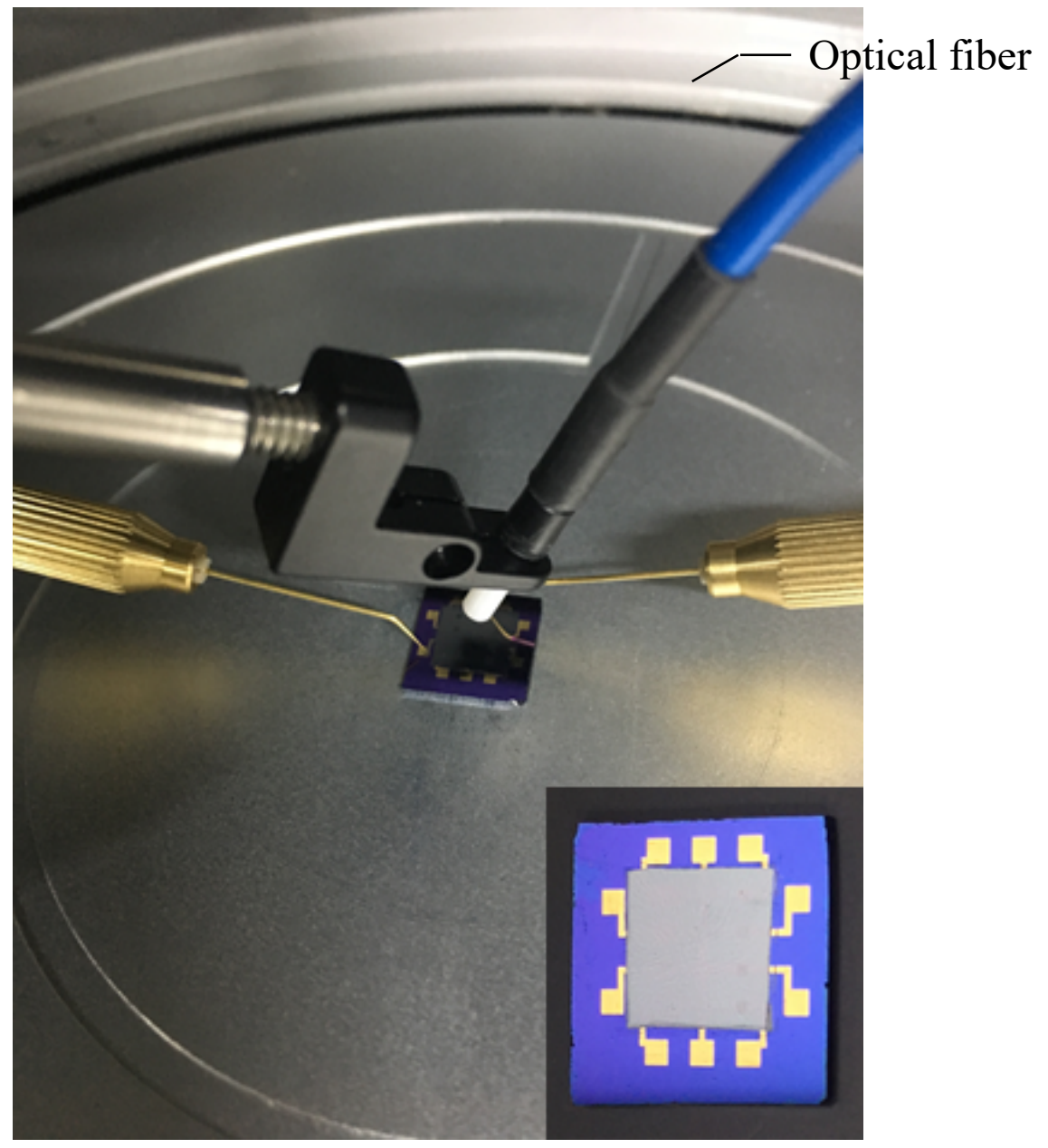

Figure S7. Two-terminal PbS QD photoconductor contacted with Au electrodes under $1310 \mathrm{~nm}$ LD illumination. The inset shows top-view of the photoconductor. 


\section{Frequency response of RTA-135 chip as a function of optical power}

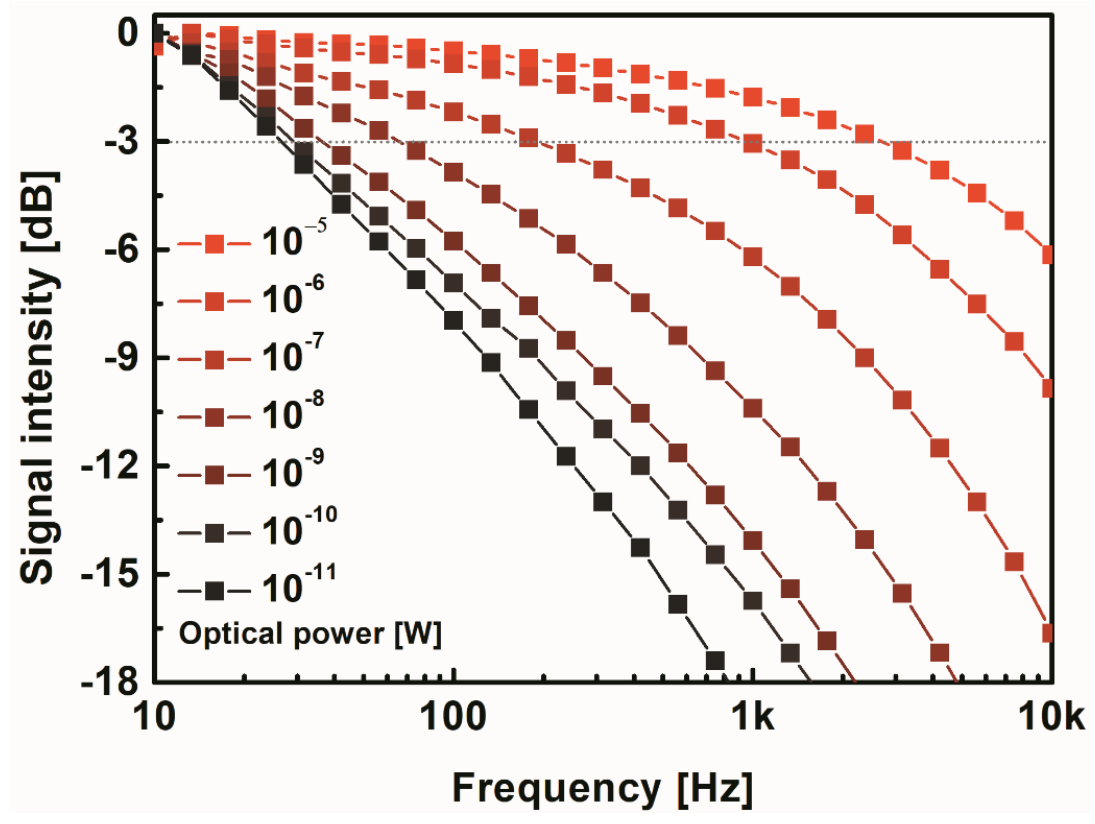

Figure S8. Frequency response of RTA-135 chip from $10 \mathrm{pW}$ to $10 \mu \mathrm{W}$. Its bandwidth decreased at low optical power.

\section{Noise characteristics of $1 \mathrm{M} \Omega$ resistor}

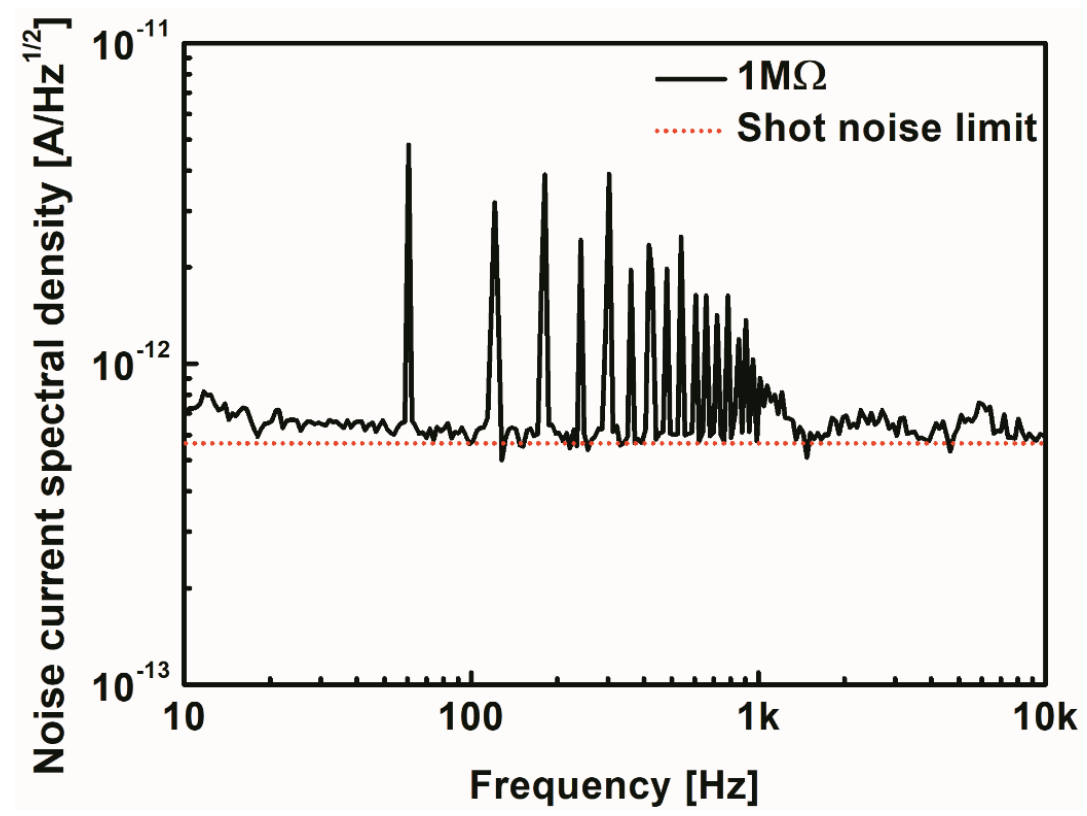

Figure S9. Noise current spectral density of $1 \mathrm{Mohm}$ resistor at $1 \mathrm{~V}$ with its shot noise limit. Peaks every $60 \mathrm{~Hz}$ are ground loop. 


\section{FT-IR spectra of $\mathrm{PbS} \mathrm{QD}$ films before and after ligand exchange}

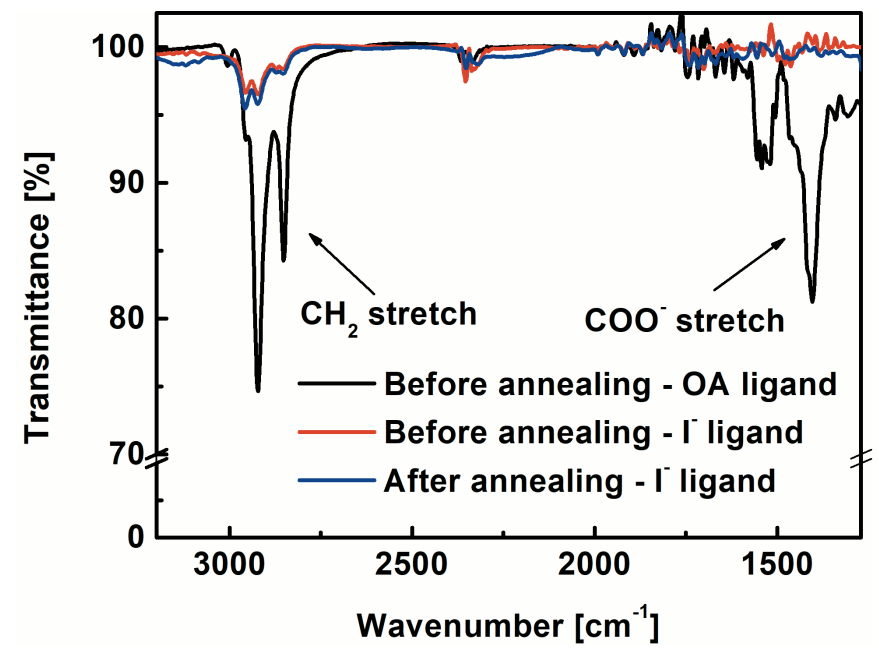

Figure S10. The FT-IR spectra of the PbS QD films with different ligands before annealing and after annealing at $135^{\circ} \mathrm{C}$. Most of the OA ligands are replaced with I- ligands. In addition, residual organic compounds are not changed after the 20 min of heat treatment.

\section{Absorbance spectra of PbS QD films}

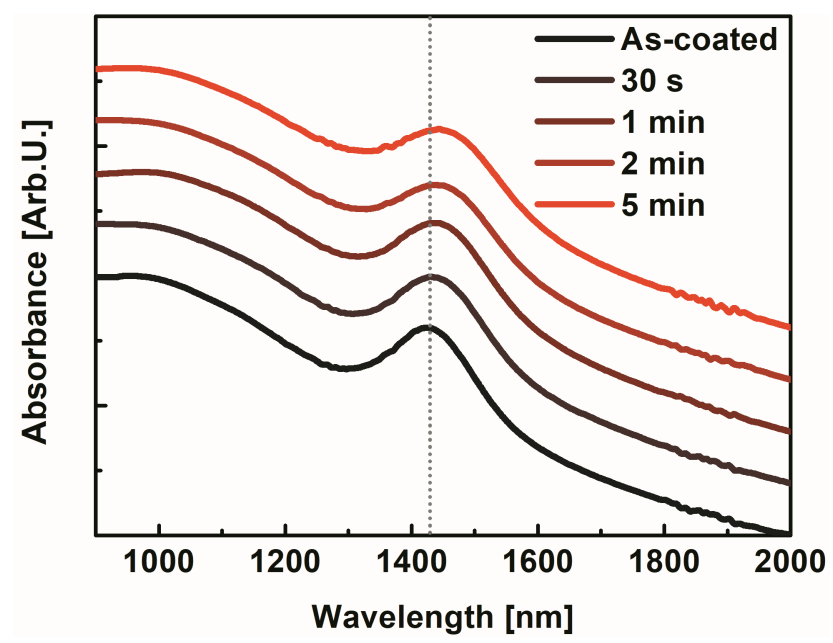

Figure S11. Absorbance spectra of PbS QD films with RTA as a function of time. The spectra indicate that the peak shifts of $\mathrm{PbS}$ QD films annealed with RTA at $135^{\circ} \mathrm{C}$ as a function of annealing time. A dotted grey line shows the 1st excitonic peak position of the as-coated film. The absorbance peaks were hardly shifted until $30 \mathrm{~s}$ annealing. The peak of the $1 \mathrm{~min}$ sample started to be red-shifted and broadened gradually until 5 min annealing. This implies that diffusion process started from $1 \mathrm{~min}$. 


\section{Grazing incidence small-angle $X$-ray scattering (GISAXS) of the annealed $\mathrm{PbS}$ QD films}

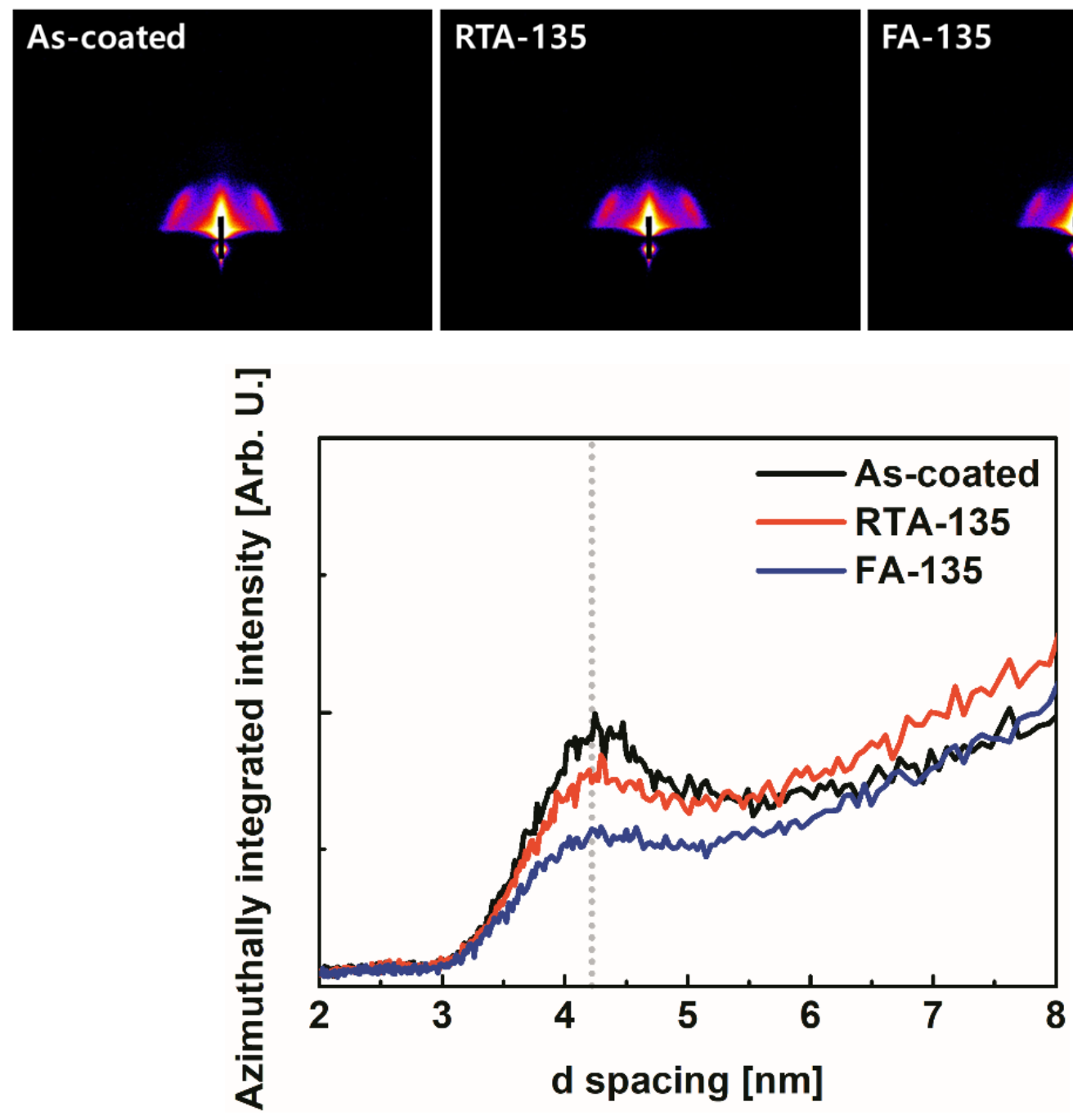

Figure S12. Grazing incidence small-angle X-ray scattering (GISAXS) patterns of the annealed QD films and their azimuthally integrated intensity. The data show no significant change in inter-particle distance between before and after thermal annealing. 


\section{Atomic force microscopy (AFM) images of the PbS QD film}
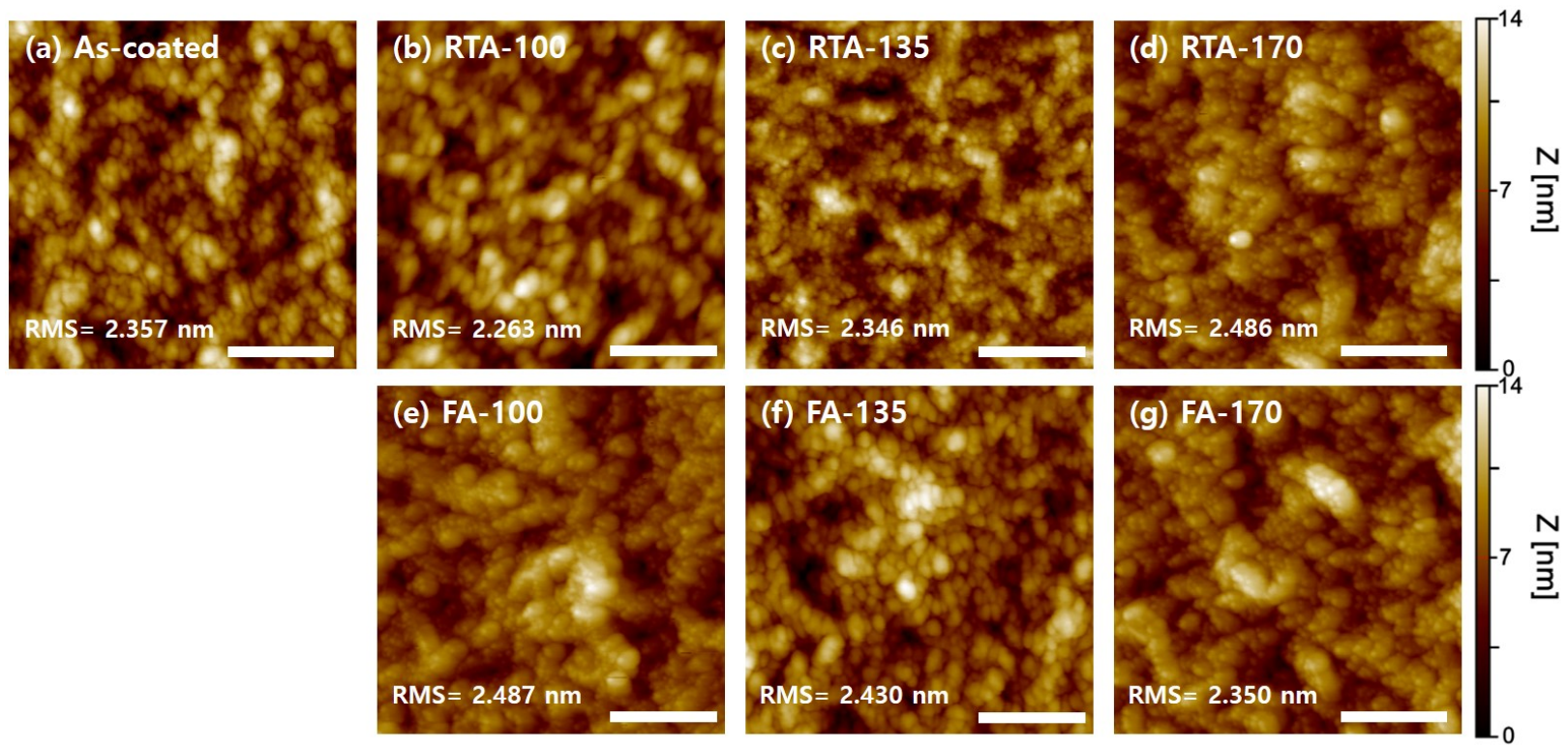

Figure S13. Atomic force microscopy (AFM) images of the $\mathrm{PbS}$ QD film before and after annealing: (a) as-coated, (b) RTA-100, (c) RTA-135, (d) RTA-170, (e) FA-100, (f) FA-135 and (g) FA-170. The films exhibit root mean square (RMS) roughness of $2.357 \mathrm{~nm}, 2.263 \mathrm{~nm}, 2.346$ $\mathrm{nm}, 2.486 \mathrm{~nm}, 2.487 \mathrm{~nm}, 2.430 \mathrm{~nm}$, and $2.350 \mathrm{~nm}$, respectively. Scale bars represents $300 \mathrm{~nm}$. The data show no significant change in RMS roughness between before and after thermal annealing. It can be attributed to low annealing temperature to sinter the QDs or decompose residual oleic acid ligand. 


\section{The specific detectivity of the photoconductor annealed with hot plate}

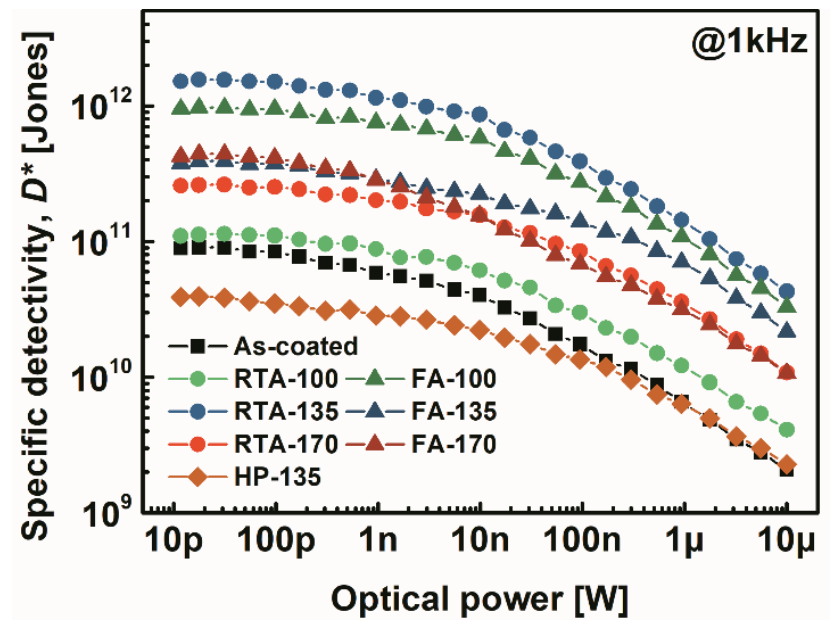

Figure S14. Specific detectivity (D*) of the photoconductors including HP-135 as a function of optical power at $1 \mathrm{kHz}$. The HP-135 sample was annealed with hot plate at $135^{\circ} \mathrm{C}$ for $30 \mathrm{~s}$. The HP-135 showed the lowest detectivity among the samples which is $3.90 \times 10^{10}$ Jones at $10 \mathrm{pW}$. It can be attributed to uneven heating or atmosphere.

\section{Standard deviation of $\mathrm{D}^{*}$}

Table S2. Standard deviation of the specific detectivity $\left(D^{*}\right)$ of the $\mathrm{PbS} \mathrm{QD}$ photoconductors from three samples, respectively.

\begin{tabular}{ccc}
\hline Sample & $\boldsymbol{D}^{*}[$ Jones] & $\begin{array}{c}\text { Standard } \\
\text { deviation [\%] }\end{array}$ \\
\hline As-coated & $8.93 \times 10^{10}$ & 19.29 \\
RTA-100 & $1.10 \times 10^{11}$ & 6.42 \\
RTA-135 & $1.51 \times 10^{12}$ & 17.4 \\
RTA-170 & $2.58 \times 10^{11}$ & 10.24 \\
FA-100 & $9.52 \times 10^{11}$ & 5.73 \\
FA-135 & $3.77 \times 10^{11}$ & 15.02 \\
FA-170 & $4.24 \times 10^{11}$ & 10.45 \\
\hline
\end{tabular}




\section{References}

(1) Manders, J. R.; Lai, T. H.; An, Y.; Xu, W.; Lee, J.; Kim, D. Y.; Bosman, G.; So, F. LowNoise Multispectral Photodetectors Made from All Solution-Processed Inorganic Semiconductors. Adv. Funct. Mater. 2014, 24, 7205-7210. https://doi.org/10.1002/adfm.201402094.

(2) Weidman, M. C.; Beck, M. E.; Hoffman, R. S.; Prins, F.; Tisdale, W. A. Monodisperse, AirStable PbS Nanocrystals via Precursor Stoichiometry Control. ACS Nano 2014, 8, 63636371. 